\section{Autoimmunity gets Mincle and dimed}

\section{By Kai-Jye Lou, Staff Writer}

Researchers at the RIKEN Research Center for Allergy and Immunology have identified MINCLE as a receptor that triggers neutrophil recruitment and secretion of proinflammatory cytokines in response to necrotic cells derived from injured tissue. ${ }^{1}$ Companies next want to see data in animals demonstrating the link between the receptor and autoimmune diseases. If the mechanistic link is confirmed, the receptor could be a more specific and selective target than the targets of currently marketed drugs.

C-type lectin domain family 4 , member e (MINCLE) falls into the class of c-type lectin receptors, which are involved in autoimmune responses. An earlier analysis of patient samples identified a correlation between MINCLE expression and rheumatoid arthritis (RA), although a causal link was not established. ${ }^{2}$ In that study, nearly half of the bone marrow-derived mononuclear cell samples from RA patients had a 5- to 50-fold increase in expression of MINCLE compared with that seen in samples from healthy controls.

In the current study, now published in Nature Immunology, the RIKEN RCAI group, led by Takashi Saito, deputy director of the center and group director of the laboratory for cell signaling, showed that an antibody against Mincle significantly lowered neutrophil infiltration in mice injected with dead melanoma or 2B4 (CD244) cells compared with what was seen in mice that received a nontargeting antibody $(p<0.05)$. 2B4 cells are members of the signaling lymphocytic activation molecule-related receptors (SRRs) that modulate NK cell activity.

The antibody against Mincle also significantly lowered neutrophil recruitment in a mouse model of radiation-induced cell death compared with what was seen in untreated or mock-treated mice $(p<0.05)$. The antibody also decreased the expression of proinflammatory cytokines secreted in response to necrotic cells, including tumor necrosis factor (TNF), IL-2 and chemokine (C-X-C motif) ligand 2 (CXCL2; MIP-2). ${ }^{1}$

\section{Defining a role}

Saito told SciBX that although Mincle is part of a class of genes associated with autoimmunity, research covering Mincle-related disease associations is still in the very early stages.

Indeed, Gordon Brown, associate professor in the Institute of Infectious Disease and Molecular Medicine at the University of Cape Town, said that "the identification of a receptor triggering inflammatory responses to necrotic cells is of great importance, and the receptor may well be involved in the development of autoimmune diseases. However, there is as yet no evidence to suggest that this receptor is involved in the development of these diseases, so speculating on its role as a potential therapeutic target is premature."

Tomas Mustelin, VP of research for inflammation at Amgen Inc., agreed. "Much work remains to be done to illuminate the possible role of MINCLE in inflammatory disease processes," he said.

Amgen markets several drugs for autoimmune diseases, including Enbrel etanercept, a soluble TNF receptor.

Bassil Dahiyat, president and CEO of Xencor Inc., thinks future work on associations between MINCLE and autoimmune disease could pay off, as the receptor's profile makes it a promising target.

"An interesting aspect of MINCLE is that it is only expressed transiently on macrophages, and expression appears to happen when the cell detects stresses like cell death," he said. "The inducible and highly regulated expression is interesting because it could be a very selective and specific target for immune modulation and not have some of the side effects seen with agents targeting more broadly expressed targets."

The downside of that transient expression, said Dahiyat, is that "the role of MINCLE might be more limited than expected."

Regardless, Dahiyat said, it is interesting and worth further study to determine the direct roles that MINCLE plays in disease processes such as RA, where Xencor's XPro 1595 TNF- $\alpha$ inhibitor is in preclinical testing.

"The etiology of cell death and tissue damage in rheumatoid arthritis is not clear, though neutrophils definitely play a role, so MINCLE is a plausible target based on the work in the paper," he said.

Brown told SciBX that compounds targeting MINCLE will need to be highly specific. "C-type lectins are relatively conserved in sequence and structure and the intracellular signaling pathways are used by many other receptors, so the therapeutic would have to specifically target MINCLE itself," he said.

\section{Next steps}

Going forward, Saito said his group plans to create and use a Mincle knockout mouse in models of autoimmune disease. The RCAI group also plans to analyze samples from patients with autoimmune diseases.

Saito also noted that his group is developing antibodies against the human version of MINCLE.

According to Dahiyat, "the next step is to examine Mincle inhibition in rodent models of arthritis as well as in other models of inflammation and autoimmunity. Perhaps most critical is verifying similar activities of human MINCLE in human cell-based assay systems, as all of the current work is being done in mice. There are often differences between human and mouse immune biology and those differences need to be carefully examined to see if human MINCLE behaves similarly."

A patent application covering the use of antibodies targeting 


\section{TARGETS \& MECHANISMS}

MINCLE and its epitopes to treat autoimmune diseases has been filed. The antibodies are available for licensing on a nonexclusive basis from the Center for Intellectual Property Strategies at RIKEN.

\section{REFERENCES}

1. Yamasaki, S. et al. Nat. Immunol.; published online Sept. 7, 2008; doi:10.1038/ni.1651

Contact: Takashi Saito, RIKEN Research Center for Allergy and Immunology, Yokohama City, Japan e-mail: saito@rcai.riken.jp

2. Nakamura, N. et al. DNA Res. 13, 169-183 (2006)

COMPANIES AND INSTITUTIONS MENTIONED Amgen Inc. (NASDAQ:AMGN), Thousand Oaks, Calif. RIKEN Research Center for Allergy and Immunology, Yokohama City, Japan

University of Cape Town, Cape Town, South Africa

Xencor Inc., Monrovia, Calif. 